ARTÍCULO DE INVESTIGACIÓN

\title{
Patogenicidad de Chrysoporthe cubensis en clones de Eucalyptus grandis y E. urophylla en el sureste de México
}

\author{
Pathogenicity of Chrysoporthe cubensis to Eucalyptus \\ grandis and E. urophylla clones in the Southeastern of Mexico
}

\author{
J. Carlos Juárez-Palacios ${ }^{1}$, J. Amador Honorato-Salazar², \\ Luis Vázquez-Silva ${ }^{1}$ y J.F. Conrado Parraguirre-Lezama ${ }^{3}$
}

\begin{abstract}
RESUMEN
Eucalyptus urophylla S.T. Blake y E. grandis Hill ex Maiden son las especies más promisorias para las plantaciones forestales en el Sureste de México. Su potencial de producción está amenazado por el patógeno del cancro, Chrysoporthe cubensis (Bruner) Gryzenhout \& M.J. Wingf., el cual afecta a la mayoría de los árboles y puede ocasionar daños de $43,6 \%$ en arbolado de $E$. grandis y hasta $40 \%$ del volumen comercial en ambas especies. Este estudio se realizó para evaluar la patogenicidad de $C$. cubensis en seis clones de E. grandis y cuatro de E. urophylla. Cuatro aislamientos de C. cubensis fueron inoculados en ramas jóvenes de los clones de ambos eucaliptos en condiciones de laboratorio y el aislamiento altamente patogénico fue seleccionado después de treinta días. Cuatro plantas de cada clon fueron subsecuentemente inoculadas en condiciones de invernadero con el aislamiento A3, que fue el más virulento, para determinar su tolerancia al patógeno después de trece meses. Los resultados mostraron que los cuatro aislamientos de $C$. cubensis son capaces de causar lesiones en las ramas jóvenes de los clones de ambas especies, observándose diferencias significativas entre los clones y los aislamientos. Todos los clones fueron susceptibles al aislamiento A3, pero mostraron niveles variables de tolerancia al ataque.
\end{abstract}

PALABRAS CLAVE:

Clones, Chrysoporthe cubensis, Eucalyptus, tolerancia, patogenicidad.

\begin{abstract}
Eucalyptus grandis S.T. Blake and E. urophylla Hill ex Maiden are the most promissory species for forest plantations in the southeastern of Mexico. Their production potential is threaten by the canker pathogen, Chrysoporthe cubensis (Bruner) Gryzenhout \& M.J. Wingf., which affects most of the trees and can cause damage of about $43,6 \%$ in $E$. grandis trees and up to $40 \%$ of the merchantable volume of both species. This study was carried out to assess the pathogenicity of $C$. cubensis in six $E$. grandis and four $E$. urophylla clones. Four isolates of $C$. cubensis were inoculated on young twigs of both eucalyptus clones in laboratory conditions and the highly pathogenic isolate was selected after thirty days. Four plants of each clone were subsequently inoculated in greenhouse conditions with the isolate $\mathrm{A} 3$, which was the most virulent, to determine their tolerance to the pathogen after thirteen months. In both trials, lesion length in the bark was measured. Results showed that the four isolates of $C$. cubensis
\end{abstract}

1 INIFAP, Campo Experimental San Martinito. CIR-Golfo Centro. Ex investigadores.

2 Autor para correspondencia. INIFAP, Campo Experimental San Martinito. CIR-Golfo Centro. Km. 56.5 Carretera Federal México-Puebla, San Martinito, Tlahuapán, Puebla. C. P. 74100. C.e.: honorato.amador@inifap.gob.mx.

3 Benemérita Universidad Autónoma de Puebla Unidad Regional Tetela. Calle Universidad s/n, Barrio Benito Juárez, Tetela de Ocampo, Puebla. 
are capable of causing lesions on young twigs of Eucalyptus clones and significant differences were observed between clones and isolates. All tested clones were susceptible to isolate $A 3$, but exhibited varying levels of tolerance to the attack.

KEY WORDS:

Clones, Chrysoporthe cubensis, Eucalyptus, tolerance, pathogenicity.

\section{INTRODUCCIÓN}

El género Eucalyptus (Myrtales: Myrtaceae), conforma una amplia diversidad biológica, con un número de especies entre 513 y 700 (Chippendale, 1988; Potts y Pederick, 2000). Diferentes especies de eucalipto se plantan en muchos lugares del mundo para la producción de madera sólida y pulpa para producción de papel, en varios países de Europa, África, Asia, América y en algunos otros lugares como las islas del pacifico, lo cual constituye un componente muy importante para la industria silvícola. En México, 202000 ha han sido plantadas con diferentes especies forestales (Conafor, 2010), de las cuales Eucalyptus urophylla S.T. Blake y Eucalyptus grandis Hill ex Maiden son las más promisorias en el sureste de México, principalmente en los estados de Chiapas, Oaxaca, Tabasco y Veracruz. La producción de estas especies es para pasta celulósica para producción de papel, madera de aserrío, pisos de parquet, postes para comunicaciones y construcción, leña y carbón vegetal (Ceccon y Martínez, 1999).

El hongo Chrysoporthe cubensis (Bruner) Gryzenhout \& M. J. Wingf. [previamente catalogado como Cryphonectria cubensis (Bruner) C. S. Hodges (Gryzenhout et al., 2004)] es causante de la enfermedad conocida como cancro del eucalipto. Este patógeno también ataca a otras especies de árboles en las áreas tropicales y sub-tropicales del mundo (Wingfield, 2003). Los daños causados por el hongo pueden ser hasta de 43,6\% en arbolado de E. grandis (Paredes et al., 2010) y mayor de $40 \%$ en el volumen de producción de madera (Venter et al., 2001), sobre todo en plantaciones comerciales.

En años recientes, numerosos aislamientos de $C$. cubensis han sido colectados de las diferentes plantaciones de eucalipto del mundo, para evaluar su patogenicidad tanto en árboles como en clones de eucaliptos (Myburg et al., 1999; Myburg et al., 2002; 2003; Gryzenhout et al., 2005).

Generalmente la patogenicidad de un organismo está influenciada por varios factores, como la temperatura, disponibilidad de nutrientes, las sequias, la defoliación y los daños mecánicos, así como aspectos genéticos, tanto del patógeno como del hospedero (Old et al., 1986; 1990; Old y Davison, 2000; Guimarães et al., 2010). En algunos casos, Chrysoporthe, está asociado con cancros muy grandes como respuesta del hospedero (Wardlaw, 1999; Nakabonge, 2006). Los cancros que se presentan anualmente en los árboles de eucalipto causan daños severos en el cambium, que pueden causar la muerte de los árboles, debido a la reacción virulenta del patógeno (Yuan y Mohammed, 2000). En otras partes del mundo, como en Sudáfrica, los cancros se desarrollan en la base de los tallos muy superficialmente, mientras que en Sudamérica y el sureste de Asia, éstos crecen a diferentes alturas de los tallos y en las ramas (Conradie et al., 1990), lo que sugiere que puede existir cierta resistencia o tolerancia al ataque del patógeno en muchos individuos.

En México, se ha encontrado el hongo de $C$. cubensis fructificando en plantas de Sericea (Clidemia: Melastomataceae), estas plantas son comunes como malezas que se encuentran en las orillas de los caminos y en las proximidades de las plantaciones de eucalipto (Ferreira, 1999). También se han encontrado estruc- 
turas reproductoras de Chrysoporthe en corteza de eucalipto en plantaciones de Chiapas y Oaxaca, que están asociadas a los cancros encontrados en la base o en otras partes de los tallos (Juárez et al., 2006; Bernal et al., 2009).

En México se han realizado pruebas de patogenicidad con algunas especies de eucalipto con el hongo clasificado anteriormente como Cryphonectria cubensis (Cruz, 2000); sin embargo, no existen reportes de este tipo de pruebas para $E$. grandis y E. urophylla, que cubren la mayor superficie de plantaciones forestales en el sureste de México, por lo que el objetivo del presente trabajo fue probar diferentes aislamientos de Chrysoporthe cubensis en clones de eucalipto para evaluar la patogenicidad del hongo y la resistencia de algún genotipo de Eucalyptus grandis y E. urophylla seleccionado y clonado.

\section{METODOLOGÍA}

\section{Selección de clones}

Los clones de E. grandis y E. urophylla, fueron obtenidos a partir de genotipos seleccionados fenotípicamente en dos ensayos de fuentes de semillas, ubicados en las localidades de Aguascalientes, Chiapas $\left(17^{\circ} 28^{\prime} \mathrm{LN}, 92^{\circ} 14^{\prime} \mathrm{LO}\right)$ y de La Gasolinera, Oaxaca $\left(17^{\circ} 20^{\prime} \mathrm{LN}, 95^{\circ} 23^{\prime}\right.$ LO) (Tabla 1). El material vegetativo fue seleccionado directamente en las plantaciones, seleccionando árboles fenotípicamente superiores y con sanidad probada visualmente. Los árboles seleccionados se derribaron dejando un tocón a una altura de $0,30 \mathrm{~cm}$ para esperar sus rebrotes. Una vez que hubo rebrotes, de cada tocón se seleccionaron ramillas tiernas de aproximadamente $0,5 \mathrm{~cm}$ de diámetro, las cuales fueron protegidas con papel secante humedecido con agua comercial de garrafón y colocadas en una hielera para evitar su deshidratación. Éstas, se llevaron a las instalaciones de la empresa Forestaciones Operativas de México, S.A. de C.V., ubicada en Las Choapas, Ver., en donde se enraizaron partes apicales de las ramas tiernas de cada fuente traída de campo, formando así los clones de cada genotipo seleccionado. Una vez enraizados y con un tamaño de altura de $50 \mathrm{~cm}$ a $60 \mathrm{~cm}$, los clones se trasladaron al invernadero del C.E. San Martinito, Puebla, del Instituto Nacional de Investigaciones Forestales, Agrícolas y Pecuarias (INIFAP), en donde se realizaron las pruebas de patogenicidad. El número total de genotipos fue de seis para E. grandis y cuatro para E. urophylla.

Los experimentos se realizaron en el laboratorio (a $19{ }^{\circ} \mathrm{C} \pm 1,5^{\circ} \mathrm{C}$ ) e invernadero $\left(a 30^{\circ} \mathrm{C} \pm 2{ }^{\circ} \mathrm{C}\right.$ ) estableciéndose dos ensayos; en el primero se realizó la inoculación de cuatro aislamientos de C. cubensis en porciones de ramas jóvenes y en el segundo la inoculación del aislamiento A3 en plantas clonadas. En cada ensayo se utilizaron cuatro plantas de clones provenientes de los genotipos seleccionados en campo, en un diseño experimental completamente al azar.

\section{Origen y selección de los aislamientos}

En las plantaciones experimentales de $E$. grandis y $E$. urophylla en Chiapas y Oaxaca, se recolectaron muestras en árboles que presentaban corteza con cancros típicos y que contenían cuerpos de reproducción del hongo. Las muestras del hongo fueron aisladas en extracto de Malta agar al $2 \%$ y fueron preservadas a $4{ }^{\circ} \mathrm{C}$, obteniéndose cuatro aislamientos $\mathrm{A} 1$ $\left(S_{2} A_{11} R_{4}\right), A 2\left(S_{3} A_{4} R_{4}\right), A 3\left(S_{3} A_{18} R_{2}\right)$ y $A 4$ $\left(\mathrm{S}_{3} \mathrm{~A}_{19} \mathrm{R}_{3}\right.$ ) de C. cubensis (Tabla 2), a los cuales se les realizó una identificación morfológica y molecular (Juárez et al., 2006; Santos et al., 2006). 
Tabla 1. Selección de clones de Eucalyptus urophylla y E. grandis de cinco fuentes establecidas en dos sitios experimentales (Aguascalientes, Chis. y La Gasolinera, Oax.) del sureste de México.

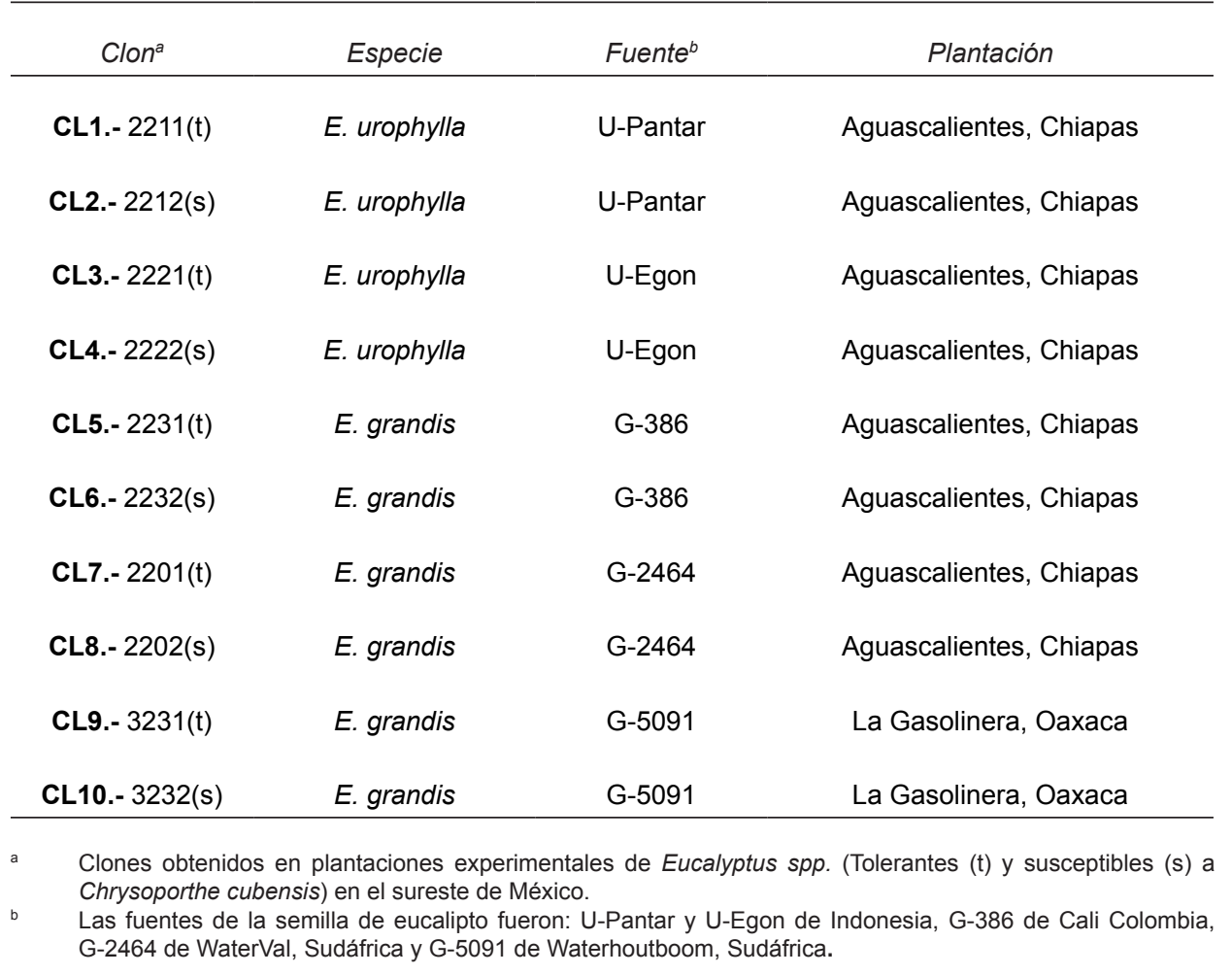

Previo a las pruebas de patogenicidad, se evaluó la agresividad de los aislamientos para lo cual se inocularon ramas de eucaliptos de 13 meses de edad, tomando como criterio de mayor agresividad del patógeno, el mayor tamaño del cancro desarrollado en las ramas inoculadas (Juárez et al., 2006).

\section{Inoculación de ramas (varetas)}

En condiciones estériles de laboratorio se inocularon cuatro ramas jóvenes de cada una de las plantas de eucalipto crecidas en invernadero. Las ramas tenían $1 \mathrm{~cm}$ de diámetro y $15 \mathrm{~cm}$ de largo que se inocula- ron con los cuatro aislamientos del hongo C. cubensis, previamente identificado (Fig. 1). Para la inoculación se hizo una perforación en la corteza de la rama con un sacabocado de $3 \mathrm{~mm}$ de diámetro en la parte central de la rama. La inoculación se realizó tomando un disco de micelio previamente desarrollado con conidias del hongo de los aislamientos seleccionados. Las ramas inoculadas se depositaron en cajas esterilizadas de plástico de $30 \mathrm{~cm} x$ $25 \mathrm{~cm} \times 20 \mathrm{~cm}$ con una rejilla y cerrado hermético, en donde se colocó agua destilada, colocándose entre la rama y el líquido una rejilla de plástico para evitar el contacto con el agua. Las cajas se colocaron en una estufa incubadora a $28^{\circ} \mathrm{C}$. La 
Tabla 2. Selección de aislamientos de Chrysoporthe cubensis, provenientes de los ensayos de fuentes de Eucalyptus del sureste de México.

\begin{tabular}{ccc}
\hline Aislamiento & Hospedero & Origen \\
\hline A1.- $\mathrm{S}_{2} \mathrm{~A}_{11} \mathrm{R}_{4}$ & E. urophylla & Aguascalientes, Chiapas \\
A2.- $\mathrm{S}_{3} \mathrm{~A}_{4} \mathrm{R}_{4}$ & E. urophylla & La Gasolinera, Oaxaca \\
A3.- $\mathrm{S}_{3} \mathrm{~A}_{18} \mathrm{R}_{2}$ & E. grandis & La Gasolinera, Oaxaca \\
A4.- $\mathrm{S}_{3} \mathrm{~A}_{19} \mathrm{R}_{3}$ & E. grandis & La Gasolinera, Oaxaca \\
\hline
\end{tabular}

$S=$ sitio, $A=a$ ábol, $R=$ repetición.

medición de los cancros se llevó a cabo con un calibrador (vernier) digital marca "Mitutoyo" Modelo CDN-P12. Las mediciones se realizaron cada cinco días en un lapso de 30 días.

\section{Inoculación en plantas jóvenes}

Para cada ensayo, se prepararon cajas de Petri (de $9 \mathrm{~cm}$ de diámetro) con extracto de Malta Agar (MYEA; $2 \%$ la malta extra y $2 \%$ agar), en donde se desarrollaron los aislamientos del hongo por 10 días a 28 ${ }^{\circ} \mathrm{C}$ creciendo en ellas colonias de micelio de color amarillo naranja.

En cuatro plantas de cada clon de eucalipto de 18 meses de edad y de 1,5 $\mathrm{cm}$ a 2,0 cm de diámetro, se inoculó el aislamiento A3 del hongo C. cubensis, identificado como el que mostró mayor agresividad en las pruebas de las varetas. Las inoculaciones se realizaron en los tallos a una altura de $20 \mathrm{~cm}$, perforando su corteza con un sacabocado de $3 \mathrm{~mm}$ de diámetro, tomando después un disco del mismo diámetro de micelio con conidias del aislamiento del hongo y colocándolo en la corteza perforada con un trozo de gasa estéril impregnada con agua destilada y fijándolo con cinta de
Parafilm "M" para evitar la pérdida de humedad (Fig. 1). Las plantas se distribuyeron al azar sobre mesas en el invernadero a una temperatura de $30^{\circ} \mathrm{C} \pm 2{ }^{\circ} \mathrm{C}$ y se regaron cada tres días. La infección del hongo y desarrollo del cancro se evaluaron cada 10 días midiendo la longitud del cancro.

\section{Análisis del desarrollo de cancros}

La caracterización del desarrollo de los cancros, ocasionados por la inoculación del hongo, se realizó con base en las variables descriptivas $c$ y $b^{-1}$ del modelo de Weibull, considerando también la severidad inicial $\left(\mathbf{Y}_{\mathrm{o}}\right)$ y final $\left(\mathbf{Y}_{\mathrm{f}}\right)$, así como el tiempo que tardó en desarrollarse el cancro como enfermedad $\left(\mathbf{T}_{\mathrm{t}}\right)$. La función de distribución de Weibull (Pennypacker et al., 1980) se seleccionó previa exploración gráfica de un total de 40 curvas de progreso inducidas por los cuatro aislamientos durante el tiempo de su desarrollo, lo cual permitió obtener un modelo flexible para describir las diversas formas de las curvas del progreso de crecimiento de los cancros. siguiente:
El modelo se expresa de la manera 


$$
Y=1-e\left[-(t / b)^{c}\right], t>0
$$

donde:

$\mathrm{Y}=$ proporción de la severidad del cancro

$\mathrm{e}=$ base del logaritmo neperiano $(2,1718)$

$\mathrm{t}=$ tiempo en días

$\mathrm{b}=$ parámetro asociado en su forma inversa con la tasa de velocidad del desarrollo del cancro

c = parámetro asociado a la forma de la curva de progreso del cancro.

El parámetro b está inversamente relacionado con la tasa de infección aparente (r) y el c está relacionado con la forma de la función de densidad $\left(\mathrm{d}_{\mathrm{y}} / \mathrm{d}_{\mathrm{t}}\right)$ de la curva de progreso del cancro. Los valores b y $\mathbf{c}$ se determinaron mediante el ajuste de regresión no lineal, usando el procedimiento NLIN del paquete estadístico SAS (SAS, Institute Inc., 1991). Después de obtenerse los valores de $b$, se obtuvo su valor inverso $\left(b^{-1}\right)$, para su uso en los análisis posteriores. Las otras variables descriptivas fueron: el tiempo total de la duración del desarrollo del cancro en la rama en días $\left(T_{t}\right)$, medido desde que se inició el desarrollo del cancro $\left(Y_{0}\right)$ hasta que éste terminó en sus mediciones.

Finalmente para conocer la diferencia estadística entre clones, agresividad de aislamientos, susceptibilidad de clones y diferencia de tiempo del desarrollo de cancros, se realizó un análisis de varianza
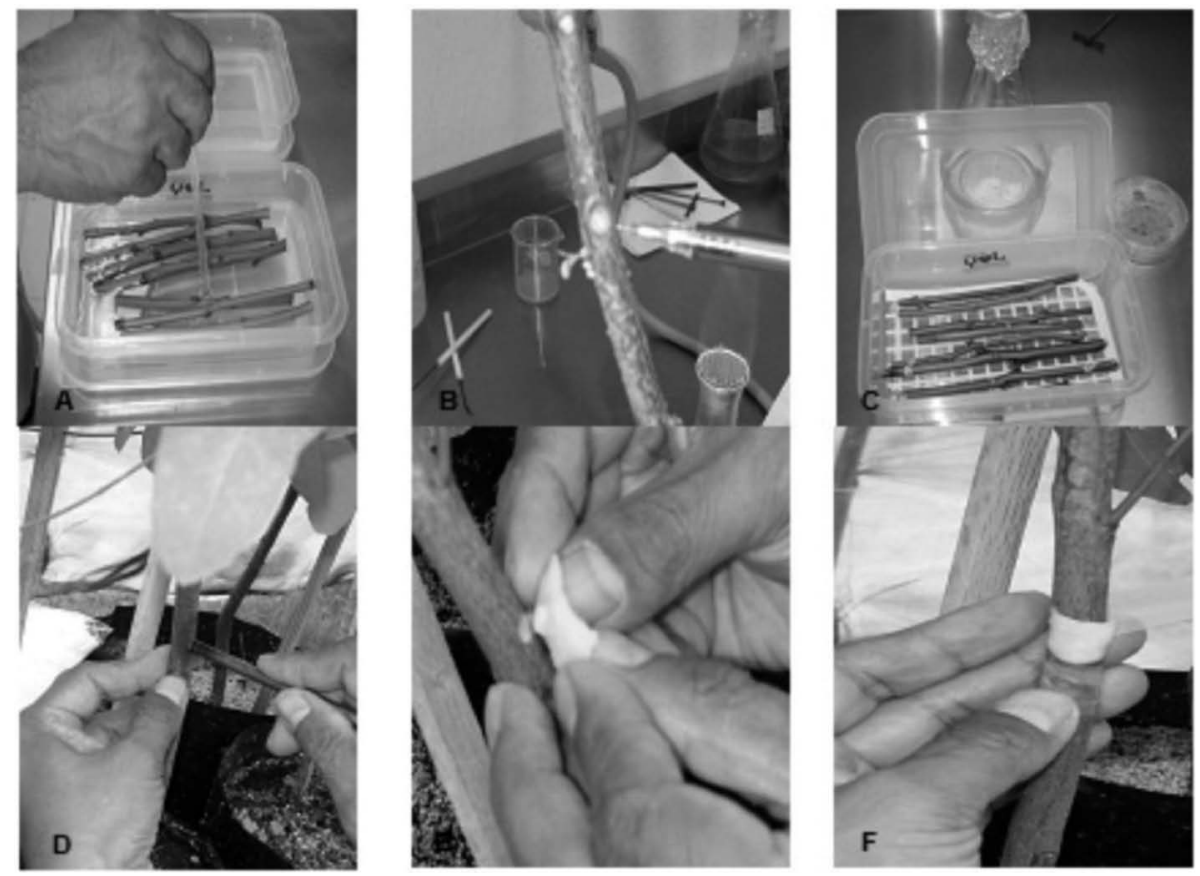

Figura 1. Inoculación de plantas de eucalipto de 18 meses de edad con los aislamientos obtenidos. A y $\mathrm{D}$ ) desinfección y perforación del tallo con sacabocados, $\mathrm{B}$ y $\mathrm{E}$ ) inóculo del hongo colocado en la perforación del tallo, C y F) cubrimiento del inóculo con gasa húmeda y Parafilm e inoculación de aislamientos en ramas y plantas de eucalipto de 1,0 $\mathrm{cm}$ de diámetro. 
de factores completamente al azar mediante el modelo general siguiente (Martínez, 1996; Wapole et al., 2007):

$$
\mathrm{y}_{\mathrm{ijkl}}=\mathrm{m}+\tau_{\mathrm{ijk}}+\varepsilon_{\mathrm{ijkl}}
$$

Siendo $y_{\mathrm{ijkl}}$ el crecimiento del cancro, $\tau_{\mathrm{ijk} \mid}$ el efecto de los factores: tiempo (i), aislamiento (j) y clon (k), para el ensayo de ramas y clon (i), resistencia (j) y etapa de desarrollo (k), para el ensayo de plantas y $\square_{i j k l}$ el error experimental. Posteriormente se realizó una comparación múltiple de medias utilizando la prueba de Tukey a un nivel de significancia de $\alpha=0,05$ (SAS, Institute Inc., 1991).

\section{RESULTADOS}

\section{Severidad de la infección en ramas de plantas jóvenes}

El desarrollo de los cancros inició a los cinco días después de la inoculación, presentando diversidad en la forma e intensidad de desarrollo de los cancros en los 10 clones evaluados. En su mayoría se encontraron curvas de desarrollo de tipo exponencial, sigmoidal, simétricas y asimétricas, mostrando diversos grados de intensidad de la enfermedad (tasas epidemiológicas); también se observaron variaciones en el inicio y la duración del desarrollo del cancro. El modelo de Weibull describió adecuadamente el desarrollo de la enfermedad a pesar de la heterogeneidad de las curvas de progreso del daño causado. Así, para los diez clones, los coeficientes de determinación $\left(R^{2}\right)$ estuvieron en un intervalo entre 0,90 y 0,98 , superando la mayoría el $90 \%$ de la variación explicada (Tabla 3). Por otra parte, en el análisis de varianza, las variables estudiadas (clon, aislamiento y tiempo de desarrollo de la lesión y su interacción), fueron altamente significativas (Tabla 4).
La mejor definición de los 10 clones de eucalipto por nivel de resistencia, fue a una distancia euclidiana promedio de 0,7 en los cuatro aislamientos evaluados y las curvas de progreso promedio de similitud de resistencia de cada grupo, se presentan en forma gráfica para cada aislamiento.

El aislamiento $A 1\left(S_{2} A_{11} R_{4}\right)$ presentó una severidad inicial $\left(Y_{0}\right)$ que fluctuó entre $0,48 \mathrm{~cm}$ y $3,53 \mathrm{~cm}$, la cual se observó a los cinco días después de la inoculación en la totalidad de los clones. La mayor severidad inicial se presentó en los clones CL2 y CL4, con valores promedios respectivos de $3,53 \mathrm{~cm}$ y $3,36 \mathrm{~cm}$. La severidad final mayor $\left(Y_{f}\right)$ a los 30 días se observó en el clon CL2, con un desarrollo de $16,04 \mathrm{~cm}$; mientras que la menor fue en el CL9 con 9,34 cm (Fig. 2). Los valores del estimador del parámetro epidemiológico $\mathrm{b}^{-1}$, de la velocidad de desarrollo del cancro, varió de 0,0025 a 0,012, el valor menor fue en el clon CL9 y el mayor fue en el clon CL1. El estimador c, tuvo valores que fluctuaron de 0,883 a 1,899, presentándose el mayor valor en el clon CL1 y en menor en el clon CL2. Los valores de ajuste $R^{2}$ en este aislamiento, variaron de 0,959 a 0,986 (Tabla 3).

Las curvas de progreso del aislamiento $A 2\left(S_{3} A_{4} R_{4}\right)$, presentó una severidad inicial $\left(Y_{0}\right)$ que fluctuó entre $0,28 \mathrm{~cm}$ a 4,02 cm, la cual se observó a los cinco días después de la inoculación en la totalidad de los clones. La mayor severidad inicial fue en el clon CL8 y la menor en el clon CL3. La severidad final mayor $\left(Y_{f}\right)$ se observó a los 30 días en el clon CL4, con un desarrollo promedio de $16,36 \mathrm{~cm}$, mientras que el clon CL3 presentó la menor severidad final con $3,49 \mathrm{~cm}$ (Fig. 3). Los valores del estimador del parámetro epidemiológico $b^{-1}$ fueron entre 0,0005 y 0,0101 ; con el menor valor para el clon CL5 y mayor para el clon CL6. 
Tabla 3. Valores promedio de las variables epidemiológicas asociadas a las curvas de progreso de la enfermedad inducida por Chrysophorte cubensis en 10 clones de eucalipto.

\begin{tabular}{|c|c|c|c|c|c|c|}
\hline Aislamiento & Clon & $c$ & $b^{-1}$ & $Y_{o}$ & $Y_{f}$ & $R^{2}$ \\
\hline \multirow{10}{*}{ A1 } & CL1 & 1,90 & 0,0120 & 0,48 & 13,41 & 0,983 \\
\hline & CL2 & 0,88 & 0,0046 & 3,53 & 16,04 & 0,963 \\
\hline & CL3 & 1,75 & 0,0113 & 0,65 & 13,97 & 0,959 \\
\hline & CL4 & 0,91 & 0,0049 & 3,36 & 15,95 & 0,962 \\
\hline & CL5 & 1,19 & 0,0055 & 1,38 & 11,07 & 0,984 \\
\hline & CL6 & 1,30 & 0,0078 & 1,47 & 14,02 & 0,986 \\
\hline & CL7 & 1,43 & 0,0080 & 0,98 & 12,07 & 0,967 \\
\hline & CL8 & 0,92 & 0,0044 & 2,98 & 14,48 & 0,975 \\
\hline & CL9 & 0,90 & 0,0025 & 1,92 & 9,34 & 0,963 \\
\hline & CL10 & 1,12 & 0,0056 & 1,81 & 12,73 & 0,966 \\
\hline \multirow{10}{*}{ A2 } & CL1 & 0,93 & 0,0021 & 1,43 & 7,40 & 0,943 \\
\hline & CL2 & 1,21 & 0,0079 & 1,98 & 16,05 & 0,975 \\
\hline & CL3 & 1,43 & 0,0032 & 0,28 & 3,49 & 0,956 \\
\hline & CL4 & 0,91 & 0,0050 & 3,43 & 16,36 & 0,955 \\
\hline & CL5 & 0,74 & 0,0005 & 1,11 & 4,13 & 0,974 \\
\hline & CL6 & 1,46 & 0,0101 & 1,28 & 16,13 & 0,969 \\
\hline & CL7 & 1,42 & 0,0055 & 0,59 & 7,35 & 0,961 \\
\hline & CL8 & 0,74 & 0,0027 & 4,02 & 14,40 & 0,935 \\
\hline & CL9 & 1,29 & 0,0048 & 0,82 & 7,88 & 0,966 \\
\hline & CL10 & 1,21 & 0,0072 & 1,78 & 14,46 & 0,946 \\
\hline \multirow{10}{*}{ A3 } & CL1 & 1,75 & 0,0112 & 0,65 & 13,87 & 0,919 \\
\hline & CL2 & 0,81 & 0,0038 & 3,98 & 15,94 & 0,926 \\
\hline & CL3 & 1,75 & 0,0116 & 0,69 & 14,58 & 0,945 \\
\hline & CL4 & 0,85 & 0,0043 & 3,80 & 16,22 & 0,956 \\
\hline & CL5 & 1,12 & 0,0056 & 1,83 & 12,77 & 0,942 \\
\hline & CL6 & 0,98 & 0,0057 & 3,09 & 16,47 & 0,925 \\
\hline & CL7 & 1,26 & 0,0078 & 1.69 & 14,96 & 0.914 \\
\hline & CL8 & 0,69 & 0,0027 & 5,13 & 16,48 & 0,939 \\
\hline & CL9 & 0,97 & 0,0042 & 2,35 & 12,62 & 0,948 \\
\hline & CL10 & 0,93 & 0,0054 & 3,41 & 16,74 & 0,926 \\
\hline \multirow{10}{*}{ A4 } & CL1 & 1,72 & 0,0103 & 0,60 & 12,45 & 0,906 \\
\hline & CL2 & 0,87 & 0,0040 & 3,24 & 14,52 & 0,968 \\
\hline & CL3 & 1,81 & 0,0112 & 0,55 & 13,03 & 0,939 \\
\hline & CL4 & 0,83 & 0,0037 & 3,54 & 14,79 & 0,937 \\
\hline & CL5 & 0,88 & 0,0022 & 1,87 & 8,69 & 0,906 \\
\hline & CL6 & 1,02 & 0,0058 & 2,71 & 15,62 & 0,904 \\
\hline & CL7 & 1,25 & 0,0057 & 1,14 & 10,28 & 0,913 \\
\hline & CL8 & 0,76 & 0,0031 & 4,09 & 15,10 & 0,921 \\
\hline & CL9 & 0,89 & 0,0027 & 2,13 & 10,15 & 0,954 \\
\hline & CL10 & 1,05 & 0,0063 & 2,61 & 15,90 & 0,940 \\
\hline
\end{tabular}

c y $\mathbf{b}^{-1}$ Forma de la curva y el estimado de la tasa de infección aparente, respectivamente, estimados por el modelo de Weibull.

$\mathbf{R}^{\mathbf{2}} \quad$ Coeficiente de determinación.

$Y_{\text {o }}$ Severidad inicial.

$\mathbf{Y}_{\mathrm{f}}$ Severidad final.

Los valores son el promedio de cuatro repeticiones. 
Tabla 4. Análisis de varianza de las variables estudiadas: clon, aislamiento y tiempo de desarrollo de la lesión ocasionada por los aislamientos del hongo C. cubensis en ramas de eucalipto.

\begin{tabular}{|c|c|c|c|c|c|}
\hline Fuente & $G L$ & $\begin{array}{c}\text { Suma de } \\
\text { Cuadrados }\end{array}$ & $\begin{array}{c}\text { Cuadrado } \\
\text { Medio }\end{array}$ & Valor de $F$ & Pr $>F$ \\
\hline Modelo & 279 & 28368,625 & 101,680 & 313,88 & $<0,0001$ \\
\hline $\mathrm{Ti}$ & 6 & 21574,519 & 3595,753 & 11099,70 & $<0,0001$ \\
\hline $\mathrm{Ai}$ & 3 & 745,640 & 248,547 & 767,24 & $<0,0001$ \\
\hline $\mathrm{CL}$ & 9 & 3167,189 & 351,910 & 1086,31 & $<0,0001$ \\
\hline Ti*Ai*CL & 261 & 2881,277 & 11,039 & 34,08 & $<0,0001$ \\
\hline Error & 840 & 272,118 & 0,324 & & \\
\hline Total Corregido & 1119 & 28640,742 & & & \\
\hline & $\mathrm{R}^{2}$ & C. V. $(\%)$ & Raíz del CME & Promedio & \\
\hline & 0,9905 & 8,88 & 0,569 & 6,41 & \\
\hline
\end{tabular}

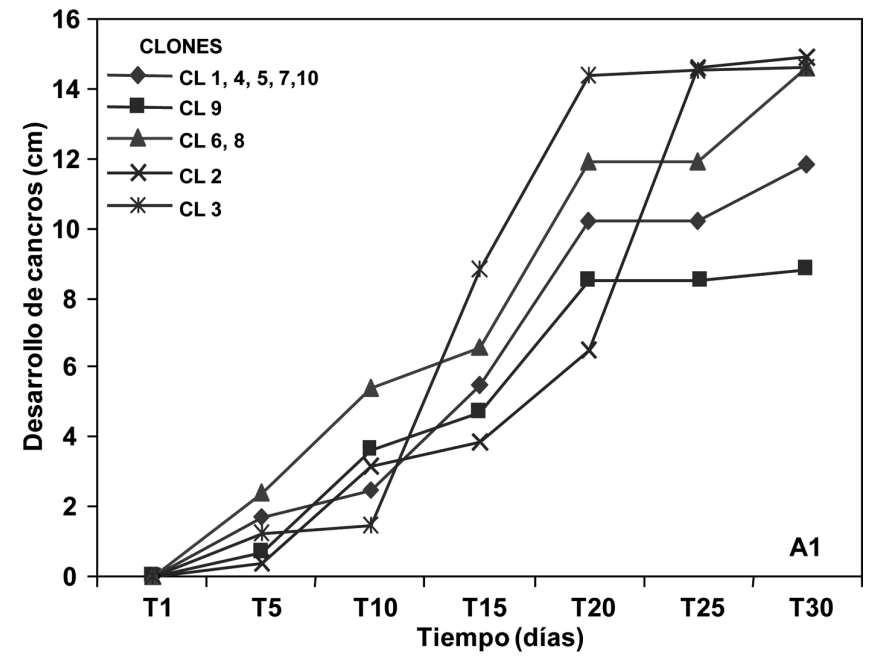

Figura 2. Curvas de desarrollo de cancros ocasionados por el aislamiento A1 del hongo Chrysoporthe cubensis. Los números de cada curva de progreso representan los nombres de los clones que tuvieron el mismo comportamiento de resistencia a la infección del hongo. 


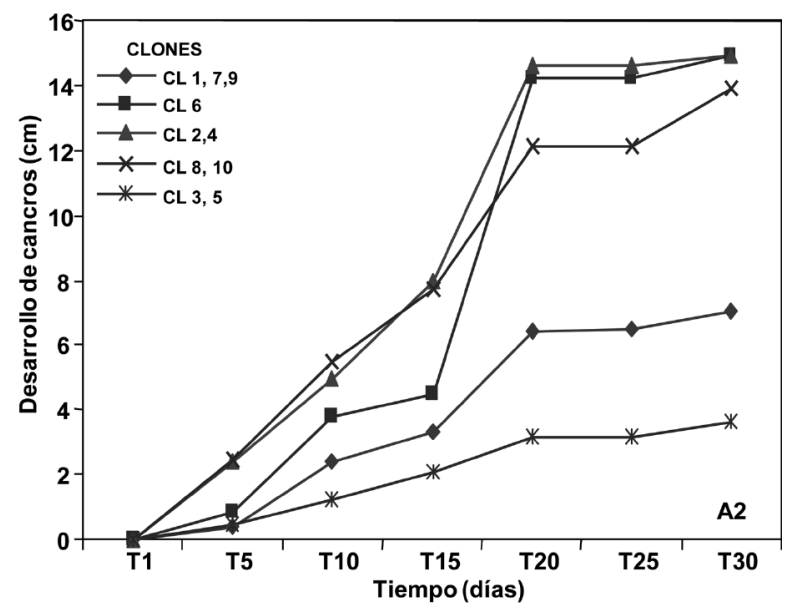

Figura 3. Curvas de desarrollo de cancros ocasionados por el aislamiento A2 del hongo Chrysoporthe cubensis.

El estimador c, tuvo valores que variaron entre 0,74 y 1,46 , que correspondieron a los clones CL5 y CL6, respectivamente. En este aislamiento, los valores de ajuste de $\mathbf{R}^{2}$ variaron de 0,943 a 0,975 (Tabla 3 ) e impactó a cinco grupos de resistencia.

Las curvas de progreso del aislamiento A3 $\left(S_{3} A_{18} R_{2}\right)$, presentaron una severidad inicial $\left(Y_{0}\right)$ que fluctuó entre $0,65 \mathrm{~cm}$ y $5,13 \mathrm{~cm}$ la cual se observó a los cinco días después de la inoculación en la totalidad de los clones, en donde la mayor severidad se presentó en el clon CL8, con un promedio de $5,13 \mathrm{~cm}$ y la menor en el clon CL1 con $0,65 \mathrm{~cm}$. La severidad final mayor $\left(Y_{f}\right)$ se manifestó a los 30 días en el clon CL10, con un desarrollo de $16,74 \mathrm{~cm}$ y la menor en el clon CL9 con 12,62 cm (Fig. 4).

Los valores del estimador del parámetro epidemiológico $\mathrm{b}^{-1}$ resultaron ser entre 0,0027 y 0,0116, siendo el menor valor para el clon CL8 y el mayor para el clon CL3. Los valores del estimador c fueron de 0,69 a 1,75, presentándose el mayor valor en el clon CL1 y el menor en el clon CL8. Los valores de ajuste de $\mathbf{R}^{2}$, para este aislamiento, fluctuaron de 0,914 a 0,956 (Tabla 3).

Las curvas de progreso del aislamiento A4 $\left(S_{3} A_{19} R_{3}\right)$, mostraron una severidad inicial $\left(Y_{0}\right)$ que fluctuó entre $0,55 \mathrm{~cm}$ a $4,09 \mathrm{~cm}$, observándose a los cinco días después de la inoculación en la totalidad de los clones. La mayor severidad inicial se presentó en el clon CL8 con un promedio de $4,09 \mathrm{~cm}$ y la menor severidad fue para el clon CL3 (Fig. 5). La mayor severidad final $\left(Y_{f}\right)$ se observó a los 30 días en el clon CL10 con un desarrollo de $15,90 \mathrm{~cm}$, mientras que la menor severidad final fue en el clon CL5. Los valores del estimador epidemiológico $b^{-1}$, de la velocidad de desarrollo del cancro, fluctuaron de 0,0022 a 0,0112, siendo el valor menor para el clon CL5 y el valor mayor para el clon CL3. Los valores de $\mathbf{c}$ fueron entre 0,76 y 1,81 , presentándose el mayor valor en el clon CL3 y en menor en el clon CL8. Los valores de ajuste de $\mathrm{R}^{2}$ en este aislamiento, variaron de 0,904 a 0,968 y se conformaron cuatro grupos de resistencia. 


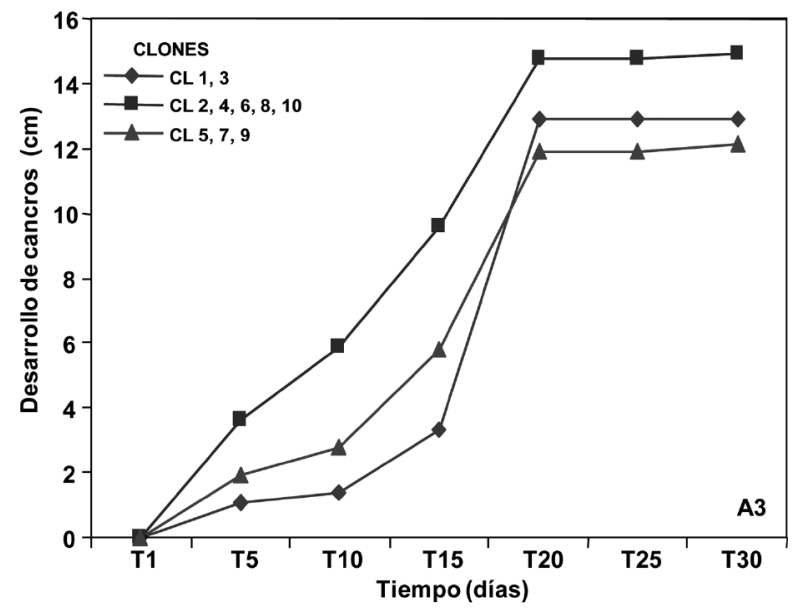

Figura 4. Curvas de desarrollo de cancros ocasionados por el aislamiento A3 del hongo Chrysoporthe cubensis.

El análisis de varianza de las ramas indicó que hubo diferencias significativas $(p \leq 0,05)$ entre clones, aislamientos y tiempo de desarrollo de los cancros (Tabla 4). La comparación de medias con la prueba de Tukey indicó que los clones se agruparon en siete grupos. Los clones
CL8 y CL4 presentaron el mayor desarrollo de cancro, mientras que el clon CL3 fue el menos afectado. Asimismo, los clones considerados como susceptibles tuvieron mayor desarrollo de cancro que los clones considerados como tolerantes (Tabla 5).

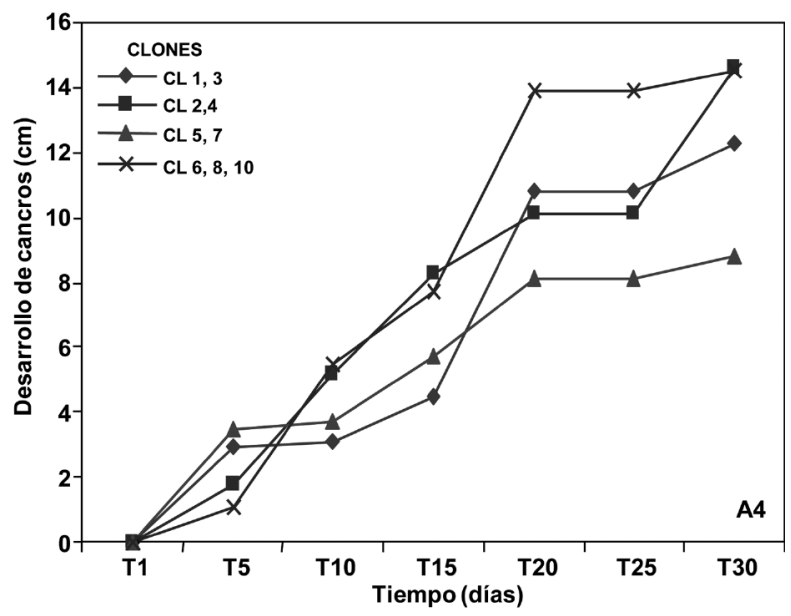

Figura 5. Curvas de desarrollo de cancros ocasionados por el aislamiento A4 del hongo Chrysoporthe cubensis. 
Tabla 5. Comparación de medias de las variables evaluadas

(clones, aislamientos y tiempo) en el desarrollo de cancro causado por el hongo

C. cubensis.

\begin{tabular}{|c|c|c|c|c|c|c|c|c|}
\hline CLONES & \multicolumn{2}{|c|}{ Promedio } & AISLAMIENTO & \multicolumn{2}{|c|}{ Promedio } & TIEMPO & \multicolumn{2}{|c|}{ Promedio } \\
\hline CL8 & 8,57 & $A$ & A3 & 7,55 & $A$ & 30 & 12,27 & A \\
\hline CL4 & 8,55 & A & A4 & 6,48 & B & 25 & 11,08 & B \\
\hline CL2 & 8,23 & B & $\mathrm{A} 1$ & 6,35 & $\mathrm{C}$ & 20 & 9,70 & C \\
\hline CL6 & 7,46 & C & $\mathrm{A} 2$ & 5,24 & $\mathrm{D}$ & 15 & 5,96 & $D$ \\
\hline CL10 & 7,43 & C & & & & 10 & 3,77 & $E$ \\
\hline CL9 & 5,09 & D & & & & 5 & 2,05 & $\mathrm{~F}$ \\
\hline CL7 & 4,93 & $E$ & & & & 1 & 0,00 & G \\
\hline CL1 & 4,81 & $E$ & & & & & & \\
\hline CL5 & 4,60 & $F$ & & & & & & \\
\hline CL3 & 4,46 & G & & & & & & \\
\hline
\end{tabular}

Variables con la misma letra no son significativamente diferentes Tukey $(\alpha=0,05)$.

Con respecto a la comparación de medias de los aislamientos, los resultados indicaron que el aislamiento A3 fue estadísticamente $(p \leq 0,05)$ diferente a los demás aislamientos y mostró mayor agresividad, seguido de los aislamientos A4, A1 y A2. El aislamiento que mostró menor agresividad fue el A2.

Hubo diferencias significativas ( $p \leq$ 0,05 ) durante el desarrollo de cancros, aumentando gradualmente con el tiempo y mostrando el mayor desarrollo a 30 días después de la inoculación.

\section{Severidad de la infección de los cancros en plantas jóvenes}

El desarrollo de los cancros inició a los 15 días después de la inoculación, 10 días más que en las ramas en condiciones controladas, presentando diversidad en la forma e intensidad de desarrollo de los cancros en los 10 clones evaluados. La dimensión de los cancros en plantas fue variable, alcanzando dimensiones de 3 $\mathrm{cm}$ a $16 \mathrm{~cm}$, por lo que en algunos casos, las plantas formaron una especie de callo en la lesión (Fig. 6. K, L). 

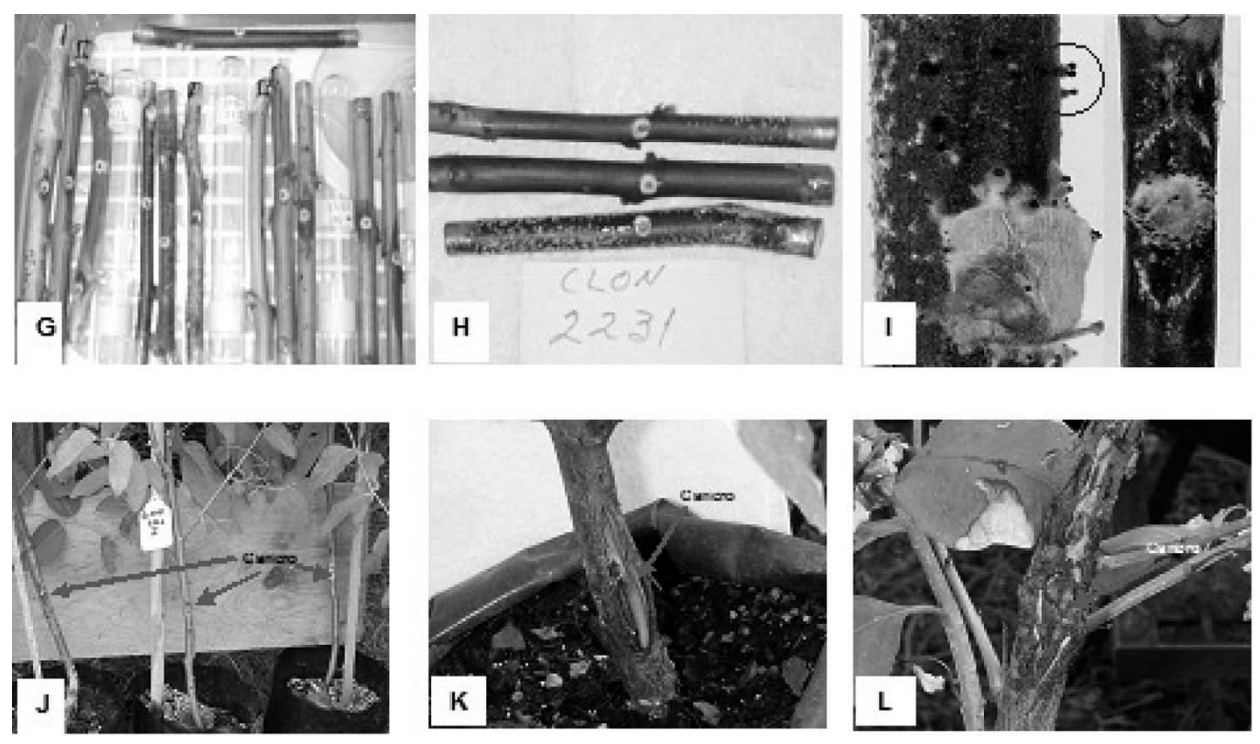

Figura 6. Dimensión de los cancros en ramas y plantas de eucalipto. ( $G$ y $L$ ) cancro definido en las ramas y tallo de la planta, $(\mathrm{H}$ y K) tallo con formación de cancro con bordes suculentos en ramas y tallo de eucalipto (I) formación de cancro con presencia de cuerpos de reproducción del hongo (picnidios), (J) formación de cancros en tallos de plantas en invernadero después de la inoculación.

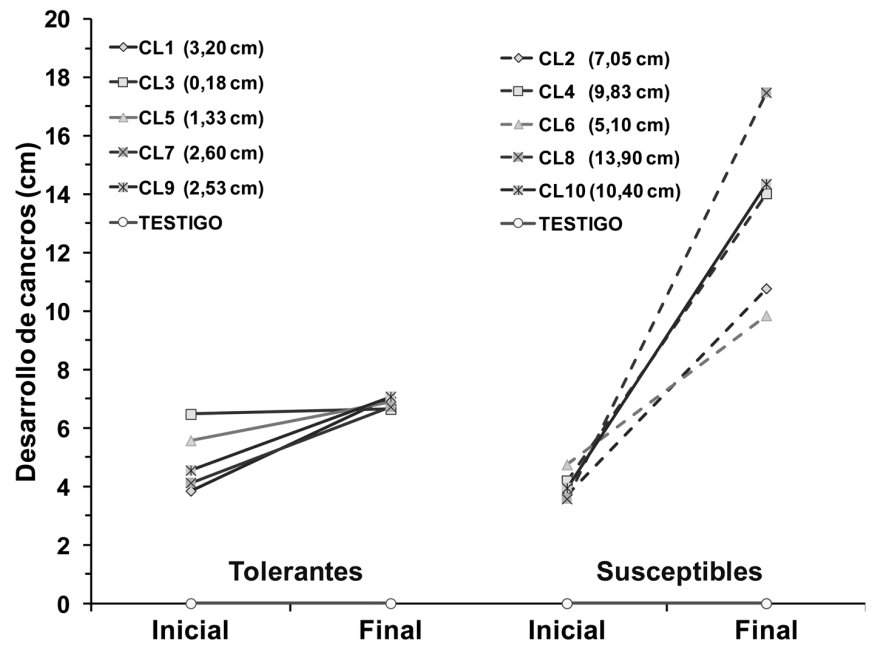

Figura 7. Desarrollo de cancros del hongo Chrisoporthe cubensis en tallos de diferentes clones de eucalipto tolerantes y susceptibles al hongo, inoculados con el aislamiento A3 a los 15 (inicial) y 395 días (final) después de la inoculación. Los valores en paréntesis son el incremento del desarrollo. 
El desarrollo del cancro inicial más bajo se presentó en el clon CL8, con un crecimiento promedio de $3,58 \mathrm{~cm}$ y el más alto en el clon CL3 con una dimensión de $6,48 \mathrm{~cm}$; mientras que en el desarrollo final del cancro, el clon CL3 mostró un crecimiento menor de $6,65 \mathrm{~cm}$ y el mayor fue en el clon CL8 con $17,48 \mathrm{~cm}$. El incremento en el desarrollo de los cancros entre el crecimiento inicial y final (después de 395 días) fue menor en los clones tolerantes que en los susceptibles. El incremento en crecimiento fue de 0,18 $\mathrm{cm}$ a 3,20 $\mathrm{cm}$ para los clones tolerantes y de $5,10 \mathrm{~cm}$ a $13,90 \mathrm{~cm}$ para los clones susceptibles (Fig. 7).
El análisis de varianza de las variables estudiadas en las plantas (clon, grupos de resistencia, etapas de desarrollo y su interacción) indicó diferencias altamente significativas ( $p \leq 0,05$, Tabla 6). El clon CL8 presentó el mayor desarrollo del cancro, mientras que el clon CL7 mostró el menor desarrollo. Al igual que en las ramas, las plantas de los clones considerados como susceptibles presentaron mayor desarrollo de cancro que las plantas de los clones considerados como tolerantes (Tabla 7). Diferencias estadísticas significativas $(p \leq 0,05)$ se encontraron entre las etapas inicial (15 días) y final (395 días).

Tabla 6. Análisis de varianza de las variables: clon (CL), resistencia (RES) y etapa (ETA), del desarrollo de la lesión ocasionada por la inoculación del aislamiento A3 del hongo C. cubensis en plantas jóvenes de eucalipto.

\begin{tabular}{|c|c|c|c|c|c|}
\hline Fuente & $G L$ & $\begin{array}{l}\text { Suma de } \\
\text { Cuadrados }\end{array}$ & $\begin{array}{l}\text { Cuadrado } \\
\text { Medio }\end{array}$ & Valor de $F$ & $\operatorname{Pr}>F$ \\
\hline Modelo & 19 & 1130,35 & 59,49 & 30,72 & $<0,0001$ \\
\hline $\mathrm{CL}$ & 9 & 223,45 & 24,83 & 12,82 & $<0,0001$ \\
\hline RES & 1 & 153,46 & 153,46 & 79,23 & $<0,0001$ \\
\hline ETA & 1 & 590,26 & 590,26 & 304,75 & $<0,0001$ \\
\hline $\mathrm{CL}^{*} \mathrm{RES}^{*} \mathrm{ETA}$ & 8 & 163,18 & 20,40 & 10,53 & $<0,0001$ \\
\hline Error & 60 & 116,21 & 1,94 & & \\
\hline \multirow[t]{3}{*}{ Total Corregido } & 79 & 1246,56 & & & \\
\hline & $\mathrm{R}^{2}$ & C. V. (\%) & Raíz del CME & Promedio & \\
\hline & 0,9068 & 19,11 & 1,392 & 7,28 & \\
\hline
\end{tabular}


Tabla 7. Comparación de medias del comportamiento de las variables evaluadas de la enfermedad cancro del eucalipto ocasionada por C. cubensis, con respecto a su desarrollo.

\begin{tabular}{|c|c|c|c|c|c|c|c|c|c|}
\hline \multirow{2}{*}{$\frac{\text { CLONES }}{\text { CL8 }}$} & \multicolumn{3}{|c|}{ Promedio } & \multirow{2}{*}{$\begin{array}{c}\text { RESISTENCIA } \\
\text { Susceptible }\end{array}$} & \multicolumn{2}{|c|}{ Promedio } & \multirow{2}{*}{$\begin{aligned} \text { ETAPA } \\
\text { Final }\end{aligned}$} & \multicolumn{2}{|c|}{ Promedio } \\
\hline & 10,53 & A & & & 8,67 & A & & 9,93 & A \\
\hline CL10 & 9,15 & B & & Tolerante & 5,90 & B & Inicial & 4,50 & B \\
\hline CL4 & 9,11 & B & & & & & & & \\
\hline CL6 & 7,30 & C & & & & & & & \\
\hline CL2 & 7,25 & C & & & & & & & \\
\hline CL3 & 6,60 & c & D & & & & & & \\
\hline CL5 & 6,24 & C & D & & & & & & \\
\hline CL9 & 5,81 & & D & & & & & & \\
\hline CL1 & 5,45 & & D & & & & & & \\
\hline CL7 & 5,43 & & D & & & & & & \\
\hline
\end{tabular}

Variables con la misma letra no son significativamente diferentes Tukey $(\alpha=0,05)$.

\section{DISCUSIÓN}

Los estudios de resistencia inducidos con diversos aislamientos fungosos de Chrysoporthe spp. en especies de eucalipto (Eucalyptus spp.) no son abundantes (e.g., Paredes et al., 2010); sin embargo, los estudios reportados indican que el eucalipto muestra una susceptibilidad relativa a la enfermedad del cancro causada por diferentes aislamientos y especies de Chrysoporthe; además muestran que la severidad de daño causado varía entre especies y aislamientos fungosos de la misma especie, siendo algunos aislamientos, de la misma especie, más patogénicos que otros (van Heerden y Wingfield, 2001, 2002; Gryzenhout et al., 2003;
Roux et al., 2003; van Heerden et al., 2005, Chen et al., 2010; Chungu et al., 2010; Guimarães et al., 2010).

Al igual que en los estudios de patogenicidad reportados, en este estudio se observó que C. cubensis es capaz de causar lesiones en clones de eucaliptos. Las lesiones causadas en las ramas y árboles jóvenes de eucalipto, en el tiempo evaluado, sugieren que la patogenicidad de $C$. cubensis puede ser capaz de causar serios daños, como ha sucedido con otras especies del patógeno (Gryzenhout et al., 2003; Chungu et al., 2010) y encontrado en plantaciones comerciales del sureste de México (Paredes et al., 2010). 
En el ensayo de pruebas de patogenicidad de aislamientos se produjeron lesiones en los 10 clones probados. La respuesta fue similar a la reportada por Chen et al. (2010) en donde diversos aislamientos de $C$. cubensis causaron daños de diferente magnitud a clones de Eucalyptus grandis. También Roux et al. (2003) y van Heerden et al. (2005) reportaron variación en las lesiones causadas por diferentes aislamientos de Cryphonectria cubensis en diversos clones de eucalipto, lo cual concuerda con los resultados obtenidos en el presente trabajo, con la diferencia de que en este estudio se utilizaron varetas para determinar la patogenicidad de los aislamientos y en los estudios reportados se utilizaron árboles de 6 a 36 meses de edad bajo condiciones de invernadero.

La longitud del daño obtenido en las varetas por aislamiento fue en promedio de $52,4 \mathrm{~mm}$ a $75,5 \mathrm{~mm}$, lo cual está dentro de los resultados reportados por Chen et al. (2010), quienes obtuvieron, después de seis semanas, un promedio de $22 \mathrm{~mm}$ a 62 $\mathrm{mm}$ para sus seis aislamientos. Sin embargo, el daño en longitud es mucho menor a lo reportado por Roux et al. (2003) para Cryphonectria cubensis, en donde se registró un daño promedio de $61,90 \mathrm{~mm}$ a $156,75 \mathrm{~mm}$ para tres aislamientos después de cuatro semanas de la inoculación.

El comportamiento variable del daño causado por el aislamiento más virulento entre los clones, es similar a los resultados obtenidos por Chen et al. (2010) para Chrysoporthe cubensis, por Conradie et al. (1992), van Heerden y Wingfield (2002) y van Heerden et al. (2005) para Cryphonectria cubensis y Gryzenhout et al. (2003) para Cryphonectria eucalypti, aunque la extensión de daño es distinto por las diferencias en los tipos de aislamientos y el tiempo de medición.

En la mayoría de los estudios de patogenicidad, el enfoque analítico ha sido univariado, esto es, que utiliza un solo parámetro de tipo epidemiológico, en la caracterización de clones o genotipos por su nivel de resistencia en condiciones de campo. Este enfoque; sin embargo, para la evaluación extensiva de clones o procedencias, no permite una completa caracterización de la resistencia (Anderson et al., 1990). En este estudio se utilizó un enfoque multivariado para caracterizar los 10 clones de eucalipto por su nivel de resistencia a los cuatro aislamientos. Este enfoque tiene como inconveniente la evaluación exhaustiva de la intensidad de la enfermedad para construir las curvas de progreso del daño y posteriormente estimar parámetros epidemiológicos asociados a estas curvas para su uso con fines comparativos (Kranz, 1983). La integración en el análisis de varios parámetros permite explicar aspectos distintos de este fenómeno, así $Y_{\text {。 }}$ permite determinar la posible existencia de la resistencia en partes de plantas jóvenes en algunos clones, mientras que la tasa de intensidad de la epidemia $\mathbf{b}^{-1}$ permite tipificar el nivel de resistencia cuantitativa. En este trabajo se decidió incluir, para fines comparativos, el análisis de conglomerados. Los parámetros de tasa epidemiológica $\mathbf{b}^{-1}$ y forma de la curva (c) fueron incluidas en estas variables por su importancia en la caracterización de la resistencia (Parlevliet y van Ommeerent, 1975).

El estudio de inoculación de aislamientos de $C$. cubensis en ramas y árboles jóvenes proporcionan datos importantes para la selección de plantas tolerantes al cancro causado por este patógeno, antes de realizar la plantación y tienen implicaciones importantes para los programas de mejoramiento de especies de eucalipto que tengan como objetivo reducir el impacto de C. cubensis. Asimismo, la inoculación artificial en invernadero es de utilidad potencial para seleccionar las plantas en un tiempo relativamente corto con un número reducido de plantas que puede 
extrapolarse a pruebas de campo (Conradie et al., 1992), por lo que se ha utilizado en pruebas de exploración para la selección de especies de eucaliptos resistentes y el manejo efectivo del cancro causado, no solo por C. cubensis, sino por otras especies (van Heerden y Wingfield, 2002; Gryzenhout et al., 2003; Roux et al. 2003; van Heerden et al., 2005; Chen et al., 2010; Guimarães et al., 2010).

\section{CONCLUSIONES}

El estudio de patogenicidad usando diferentes aislamientos de Chrysoporthe cubensis en diferentes clones de Eucalyptus grandis y $E$. urophylla del Sureste de México indicó diferentes grados de tolerancia al patógeno entre los clones, tanto en ramas como en los árboles jóvenes.

Los clones susceptibles CL8 (Eucalyptus grandis) y CL4 (E. urophylla) de las fuentes G-2464 y U-Egon, respectivamente, fueron los más afectados por el ataque de Chrysoporthe cubensis en ramas y plantas jóvenes.

El modelo de Weibull describió adecuadamente el desarrollo de la enfermedad considerando la severidad inicial $\left(\mathbf{Y}_{\mathrm{o}}\right)$ y final $\left(\mathbf{Y}_{\mathrm{f}}\right)$, la tasa de infección aparente $\left(\mathbf{b}^{-1}\right)$, el valor de la forma de la curva (c) y el tiempo de desarrollo del cancro $\left(\mathbf{T}_{\mathbf{t}}\right)$.

Los clones inoculados, tanto en las varetas como en los árboles jóvenes mostraron diferencias en su nivel de tolerancia al daño causado por C. cubensis, siendo mayor el daño en los clones susceptibles que en los clones tolerantes.

\section{RECONOCIMIENTOS}

Agradecemos los apoyos y facilidades brindadas para realizar el estudio a la empresa Forestaciones Operativas de
México, S.A. de C. V. de las Choapas, Ver y al Fondo Sectorial Conafor-Conacyt por los apoyos económicos para el proyecto Conacyt-Conafor 2004-CO4-41. Asimismo, agradecemos las observaciones y correcciones realizadas por los árbitros para mejorar el presente trabajo.

\section{REFERENCIAS}

Anderson, W.F., M.K. Beute, J.C. Wynne y S. Wonkaew. 1990. Statistical procedures for assessment of resistance in multiple foliar disease complex of peanut. Phytopathology 80:14511459.

Bernal, L.I.O., J.C. Juárez P., R. Santos G., L. Vázquez S., H.A. Zavaleta M., H.S. Azpiroz R., J.F.C. Parraguirre L. y M.P. De la Garza L.L. 2009. Identificación del agente patógeno del cancro del eucalipto en plantaciones del sureste de México. Ciencia Forestal en México 34 (105): 19-37.

Ceccon, E. y M. Martínez R. 1999. Aspectos ambientales referentes al establecimiento de plantaciones de eucalipto de gran escala en áreas tropicales: aplicación al caso de México. Interciencia 24 (6):352-359.

Chen, S.F., M. Gryzenhout, J. Roux, Y.J. Xie, M.J. Wingfield y X. D. Zhou. 2010. Identification and pathogenicity of Chrysoporthe cubensis on Eucalyptus and Syzygium spp. in South China. Plant Disease 94:1143-1150.

Chippendale, G. M. 1988. Flora of Australia Volume 19 - Myrtaceae - Eucalyptus Angophora. Australian Government Publishing Service, Canberra, Australia. $542 \mathrm{p}$.

Chungu, D., M. Gryzenhout, A. MuimbaKankolongo, M.J. Wingfield y J. 
Roux. 2010. Taxonomy and pathogenicity of two novel Chrysoporthe species from Eucalyptus grandis and Syzygium guineense in Zambia. Mycological Progress 9 (3):379-393.

Comisión Nacional Forestal (Conafor). 2010. Superficie Establecida Plantaciones Forestales Comerciales. Prodeplan/Padrones de Consulta. http:// www.conafor.gob.mx/portal/index. php/temas-forestales/plantaciones. (Consultado, Febrero 20, 2012).

Conradie, E., W.J. Swart y M.J. Wingfield. 1990. Cryphonectria canker of Eucalyptus, an important diseases in plantation forestry in South Africa. South African Forestry Journal 152:43-49.

Conradie, E., W.J. Swart y M.J. Wingfield. 1992. Susceptibility of Eucalyptus grandis to Cryphonectria cubensis. European Journal of Forest Pathology 22:312-315.

Cruz, A.J. 2000. Etiología y síndrome de los cancros Botriodiplodia, Cryphonectria y Fusicoccun en plantas y varetas de eucalipto (Eucalyptus camaldulensis Dehnh). Tesis de Maestro en ciencias Forestales. Universidad Autónoma de Chapingo, Edo. De México. 224p.

Ferreira, F.A., A.M. Demuner, N.L. Demuner y S. Pigatto. 1999. Murcha-deCeratocystis em eucalipto no Brasil. (Abstr.) Fitopatologia Brasileira 24:284.

Gryzenhout, M., H. Myburg, B.D. Wingfield, F. Montenegro y M.J. Wingfield. 2005. Chrysoporthe doradensis sp. nov. pathogenic to Eucalyptus in Ecuador. Fungal Diversity 20:39-57.

Gryzenhout, M., H. Myburg, N.A. Van der Merwe, B.D. Wingfield y M.J. Wing- field. 2004. Chrysoporthe, a new genus to accommodate Cryphonectria cubensis. Studies in Mycology 50:119-142.

Gryzenhout., M, B.E. Eisenberg, T.A. Coutinho, B.D. Wingfield y M.J. Wingfield. 2003. Pathogenicity of Cryphonectria eucalypti to Eucalyptus clones in South Africa. Forest Ecology and Management 176:427-437.

Guimarães, L.M.S., M.D.V. Resende, D. Lau, L.N. Rosse, A.A. Alves y A.C. Alfenas. 2010. Genetic control of Eucalyptus urophylla and E. grandis resistance to canker caused by Chrysoporthe cubensis. Genetics and Molecular Biology 33(3):525-531.

Juárez, P.J.C., L. Vázquez S., I.O. Bernal L., R. Santos G. y S.H. Azpiroz R. 2006. Aislamiento e identificación morfológica del cancro del eucalipto Crysoporthe cubensis (Bruner) Hodges en plantaciones de Chiapas y Oaxaca. In: Instituto Nacional de Investigaciones Forestales, Agrícolas y Pecuarias. Memorias de la Reunión Nacional de Investigación Forestal. Publicación Especial núm. 1, CENID-COMEF, Coyoacán D.F. $\mathrm{p}: 86-87$.

Kranz, J. 1983. Epidemiological Parameters of Plant Resistance. In: Lamberti, F., J. M. Waller y N. A. van der Graff, eds. Durable Resistance in Crops. Plenum Press, Nueva York. p:141-161.

Martínez, G.A. 1996. Diseños experimentales: métodos y elementos de teoría. Reimp. Editorial Trillas. México, D. F. 756 p.

Myburg, H., B.D. Wingfield y M.J. Wingfield. 1999. Phylogeny of Cryphonectria cubensis and allied species 
inferred from DNA analysis. Mycologia 91:243-250.

Myburg, H., M. Gryzenhout, B.D. Wingfield y M.J. Wingfield. 2003. Conspecificity of Endothia eugeniae and Cryphonectria cubensis: a re-evaluation based on morphology and DNA sequence data. Mycoscience 104:187-196.

Myburg, H., M. Gryzenhout, R.N. Heath, J. Roux, B.D. Wingfield y M.J. Wingfield. 2002. Cryphonectria canker on Tibouchina in South Africa. Mycological Research 106:1299-1306.

Nakabonge, G., J. Roux, M. Gryzenhout y M.J. Wingfield. 2006. Distribution of Chrysoporthe canker pathogens on Eucalyptus and Syzygium spp. in eastern and southern Africa. Plant Disease 90:734-740.

Old, K.M. y E.M. Davison. 2000. Canker diseases of eucalypts. In: Keane, P.J., G.A. Kile, F.D. Podger y B.N. Brown, eds. Diseases and pathogens of eucalypts. CSIRO Publishing. Collingwood, Australia. p:214-257.

Old, K.M., D.I.L. Murray, G.A. Kile, J. Simpson y K.W.J. Malafant. 1986. The pathology of fungi isolated from eucalypt cankers in south-eastern Australia. Australian Forest Research 16:21-36.

Old, K.M., R. Gibbs, I. Craig, B.J. Myers y Z. Q. Yuan. 1990. Effect of drought and defoliation on the susceptibility of eucalypts to cankers caused by Endothia gyrosa and Botryo-sphaeria ribis. Australian Journal of Botany 38:571-581.

Paredes-Díaz E., J. Jasso-Mata, J. LópezUpton y D. Alvarado-Rosales. 2010. Presencia de Chrysoporthe cubensis
(Bruner) Gryzenhout \& M.J. Wingf. en plantaciones de Eucalyptus grandis Hill Ex Maiden en Oaxaca, México. Revista Mexicana de Ciencias Forestales. 1(2):81-92.

Parlevliet, J.E. y A. van Ommeerent. 1975. Partial resistance of barley to leaf rust, Puccinia hordei II. Relationship between field trial, microplot test and latent period. Euphytica 24:296-303.

Pennypacker, S.P., H.D. Knoble, C.E. Antle y L.V. Madden. 1980. A flexible model for studying plant disease progression. Phytopathology 70:232235.

Potts, B.M. y L.A. Pederick. 2000. Morphology, phylogeny, origin, distribution and genetic diversity of the eucalypts. In: Keane, P.J., G.A. Kile, F.D. Podger y B.N. Brown, eds. Diseases and pathogens of eucalypts. CSIRO Publishing, Melbourne, Australia. p:11-34.

Roux, J., H. Myburg, B.D. Wingfield y M.J. Winfield. 2003. Biological and phylogenetic analyses suggest that two Cryphonectria spp. cause cankers of Eucalyptus in Africa. Plant Disease 87(11):1329-1332.

Santos, G.R., J.C. Juárez P., H.S. Azpiroz R., L.Vazquez S. e I.O. Bernal L. 2006. Identificación de Crysophorte spp. (Gryzenhout and M. J. Wingf) procedentes de plantaciones de Eucalyptus spp. In: Instituto Nacional de Investigaciones Forestales, Agrícolas y Pecuarias. Memorias de la Reunión Nacional de Investigación Forestal. Publicación Especial núm. 1, CENID-COMEF, Coyoacán D.F. $\mathrm{p}: 45-46$.

SAS Statistical Software, 1991. SAS/STAT User's Guide, Version 6, Fourth Edi- 
tion, Vol. 1 \& 2. SAS Institute Inc., Cary, NC, EUA.

van Heerden, S.W. y M.J. Wingfield. 2001. Genetic diversity of Cryphonectria cubensis isolates in South Africa. Mycological Research 105:94-99.

van Heerden, S.W., y M.J. Wingfield. 2002. Effect of environment on the response of Eucalyptus clones to inoculation with Cryphonectria cubensis. Forest Pathology 32:395402.

van Heerden, S.W., H.V. Amerson, O. Preisig, B.D. Wingfield, y M.J. Wingfield. 2005. Relative pathogenicity of Cryphonectria cubensis on Eucalyptus clones differing in their resistance to C. cubensis. Plant Disease 89:659662.

Venter, M., M.J. Wingfield, T.A. Coutinho y B.D. Wingfield. 2001. Molecular characterization of Endothia gyrosa isolates from Eucalyptus in South Africa and Australia. Plant Pathology 50:211-217.

Wapole, R.E., R.H. Myers, S.L. Myers y K. Ye. 2007. Probability \& statistics for engineers \& scientists. Pearson Prentice Hall, Bostos, MA, EUA. 791 p.

Wardlaw, T.J., 1999. Endothia gyrosa associated with severe stem cankers on plantation grown Eucalyptus nitens in Tasmania, Australia. European Journal of Forest Pathology 29:199-208.

Wingfield, M.J. 2003. Increasing threat of diseases to exotic plantation forests in the southern hemisphere: lessons from Cryphonectria canker. 2003 Daniel McAlpine Memorial Lecture. Australasian Plant Pathology 32:133-139.

Yuan, Z.Q. y C. Mohammed. 2000. The pathogenicity of isolates of Endothia gyrosa to Eucalyptus nitens and E. globulus. Australasian Plant Pathology 29:29-35. cidad de Chrysoporthe cubensis en clones de Eucalyptus grandis y E. urophylla en el sureste de México. Madera y Bosques 19(1):17-36. 